\title{
Metachronous Double Primary Gastric and Colorectal Cancer: Is Prognosis Better with Gastric or Colorectal Cancer Occurring First?*
}

\author{
Mitsugu Kochi, Masashi Fujii, Noriaki Kanamori, Yoshiaki Mihara, Tomoya Funada, \\ Hidenori Tamegai, Megumu Watanabe, Yuriko Takayama, Hiroshi Suda, Tadatoshi Takayama\#
}

Department of Digestive Surgery, Nihon University School of Medicine, Tokyo, Japan.

Email: "kochi.mitsugu@nihon-u.ac.jp

Received February $12^{\text {th }}, 2013$; revised March $14^{\text {th }}, 2013$; accepted March $21^{\text {st }}, 2013$

Copyright (C 2013 Mitsugu Kochi et al. This is an open access article distributed under the Creative Commons Attribution License, which permits unrestricted use, distribution, and reproduction in any medium, provided the original work is properly cited.

\begin{abstract}
The purpose of this study was to investigate the clinicopathological features of gastric precedence (GP) and colorectal precedence (CP) metachronous double primary gastric and colorectal cancer (MDPGCC) and determine the biological significance of these two types of malignancy in making a prognosis. Between January 1990 and December 2009, 4523 patients underwent surgical treatment or chemotherapy, but no endoscopic resection for gastric or colorectal cancer. From this group, we selected those patients in whom another gastric or colorectal primary cancer developing from another origin had been diagnosed. For classification as MDPGCC there had to be an interval of 6 months or more before a secondary diagnosis of gastric or colorectal cancer. Among 4523 patients treated for gastric or colorectal cancer, MDPGCC was diagnosed in 54 patients (1.2\%). The selected patients were classified into a GP ( $\mathrm{n}=30)$ or CP group ( $\mathrm{n}$ $=24)$. No statistically significant differences were observed between the two groups with regard to sex, age, operation, location or histological type. No differences were observed in rates of surgery between the two groups. No notable difference was observed in the year-by-year incidence of GP- and CP-MDPGCC as calculated from the date of surgery or chemotherapy for the secondary gastric or colorectal cancer. The 5-year survival rate in the GP- and CP-MDPGCC groups was $84.7 \%$ and $83.3 \%$, respectively. No significant difference was observed between the GP- and CP-MDPGCC groups $(\mathrm{P}=0.9)$. There is no significant difference in prognosis between GP- and CP-MDPGCC.
\end{abstract}

Keywords: Colorectal Cancer; Gastric Cancer; Metachronous Double Primary Cancer; Prognosis

\section{Introduction}

The leading cause of death in Japan was recently reported to be malignant tumors, with the incidence of colorectal cancer showing a rapid increase, probably due to environmental changes, a rapidly aging society and the increasing westernization of lifestyle and dietary habits $[1,2]$. On the other hand, great improvement in length of survival in cancer patients has been achieved through remarkable advances in cancer treatment. One problem, however, is the danger of the cancer metastasizing and the patient subsequently dying from a secondary cancer. The incidence of double primary gastric and colorectal

${ }^{*}$ Conflict of Interest Statement: M. Kochi, M. Fujii, N. kanamori, Y. Mihara, T. Funada, M. Watababe, Y. Takayama, Hiroshi Suda and T. Takayama, all of the authors declare that they have no conflict of interest in relation to this work.

${ }^{\#}$ Corresponding author. cancer (DPGCC), both synchronous (SDPGCC) and metachronous (MDPGCC), has increased with the concomitant increase in the prevalence of gastric and colorectal cancers in Japan. Some studies on double primary cancer in patients with gastric cancer have reported that synchronous cancer had a worse prognosis than metachronous cancer [3-6]. We reported that SDPGCC had a worse prognosis than MDPGCC in patients with gastric and colorectal cancer. 7 However, with MDPGCC it remains to be established whether the prognosis is better with prior incidence of gastric cancer (gastric precedence, $\mathrm{GP}$ ) or colorectal cancer (colorectal precedence, CP). Further characterization of MDPGCCs would provide valuable information for the early diagnosis and treatment of these diseases. The purpose of this study was to investigate the clinicopathological features of GP- and CP-MDPGCC and determine the biological significance of these two types of malignancy in making a prognosis. 


\section{Materials and Methods}

\subsection{Patients}

Between January 1990 and December 2009, 4523 patients underwent surgical treatment or chemotherapy, but no endoscopic resection for primary gastric or colorectal cancer at Nihon University Medical Hospital.

\subsection{Surgical Procedure}

Among 2162 patients with primary gastric cancer, surgical resection was performed in 1905 (total gastrectomy in 431 and partial resection in 1474) and chemotherapy in 257. Among 2361 patients with colorectal cancer, surgical resection was performed 2169 (1388 with colon cancer and 781 with rectal cancer) and chemotherapy in 192.(Figure 1) Indications for endoscopic resection in all colorectal cancer patients from 1990 comprised endoscopic mucosal resection (EMR) or polypectomy where mucosal or one-third submucosal invasion (sm-1) was less than $2 \mathrm{~cm}$; from 2005, this was also indicated for gastric cancer where the depth of invasion was less than $2 \mathrm{~cm}$ and the pathological diagnosis was differentiated type. These procedures were not performed in such cases before 2005. In cases that did not fall into this category, surgical resection was performed in those which were curative and chemotherapy in those which were noncurative with distant metastases. From this group, we selected those patients in whom another gastric or colorectal primary cancer developing from another origin had been surgically resected at this or another hospital.

\subsubsection{MDPGCC Classification}

For classification as MDPGCC, there had to be an interval of 6 months or more between a secondary diagnosis of gastric or colorectal cancer and resection or chemotherapy for colorectal or gastric cancer, respectively. An interval of 6 months or more was selected as this is the period most often indicated in the literature on meta- chronous cancers of the digestive tract and screening is carried out at these intervals at our institute.

\subsubsection{MDPGCC Patients Characteristics}

Among 4523 patients treated for gastric or colorectal cancer, MDPGCC was diagnosed in 54 patients. Gastrectomy was performed in all 22 patients who had undergone colorectal resection for colorectal cancer (total, 5; partial, 17). Colorectal resection was performed in 28 out of 30 patients who had undergone gastrectomy (colon, 22; rectum, 6) (Figure 1). Median age was 68 years (37 - 81 years). The cohort included 40 men and 14 women. Patients were classified into 2 groups according to cancer detection as follows: GP-MDPGCC or CPMDPGCC. No evidence of hereditary disease was found in any patient. All gastric or colorectal cancer patients underwent endoscopy of the upper digestive tract and colonoscopy as part of their routine preoperative gastric and colorectal cancer work-up. The histological diagnosis was established by gastrointestinal endoscopic biopsy in all cases. Staging was established by a complete physical examination, complete blood count and biochemical profile, gastrointestinal contrast study, chest and abdominal computed tomography (CT), and preoperative abdominal ultrasound examination. No adjuvant chemotherapy was given.

\subsubsection{Chemotherapy of Non-Resectable MDPGCC Patients}

Among patients with primary colorectal cancer, chemotherapy was carried out in 2 with non-resectable gastric cancer (1 case, 5-fluorouracil (5FU) + cisplatin (FP); 1 case, S-1 + Docetaxel) and 2 (FP) with recurrent gastric cancer. Among patients with primary gastric cancer, chemotherapy was carried out in 2 with non-resectable colorectal cancer (1 case, FOLFOX; 1 case, 5FU + loucovorin $(\mathrm{LV})$ ) and 3 with recurrent colorectal cancer $(5 \mathrm{FU}+\mathrm{LV})$.

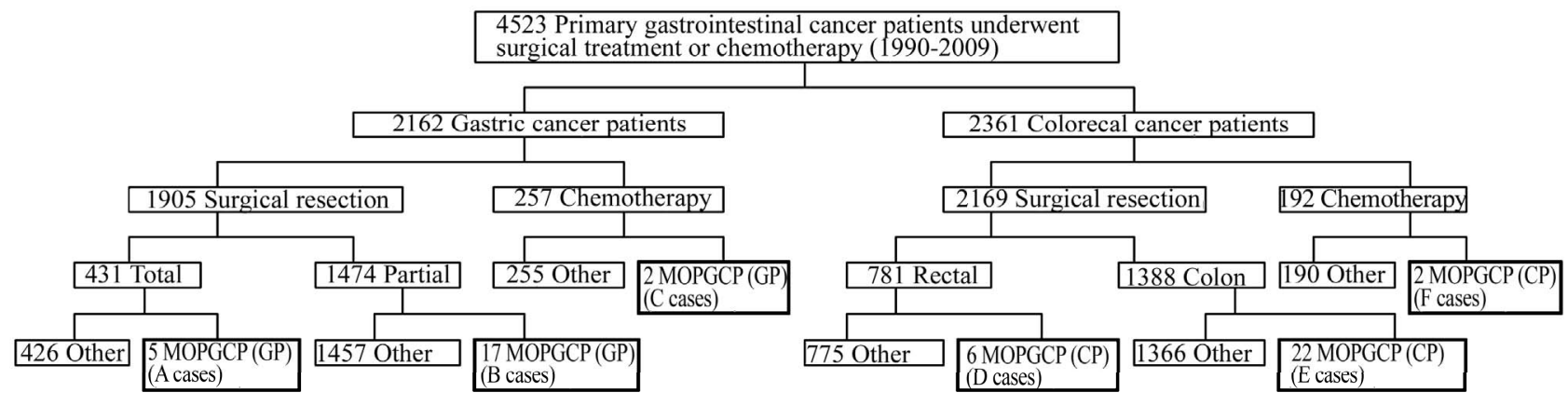

$\operatorname{MDPGCP}(\mathrm{GP}) \mathrm{A}+\mathrm{B}+\mathrm{C}=30$

$\operatorname{MDPGCP}(\mathrm{CP}) \mathrm{D}+\mathrm{E}+\mathrm{F}=24$

Figure 1. Distribution of the patients. 


\subsection{Statistical Analysis}

Overall survival curves were generated by the KaplanMeier method and between-group differences were compared by the log-rank test. The survival curves in the GPand CP-MDPGCC groups were calculated from the date of surgery or chemotherapy for the second gastric or colorectal cancer. A P value of less than 0.05 was considered to indicate statistical significance. SAS for Windows version 8.02 (SAS Institute Inc., USA) and Microsoft Excel 2003 (Microsoft Co., Ltd., Japan) were used for the statistical analysis and data calculation.

\section{Results}

\subsection{Clinicopathological Features}

Among 4523 patients treated for gastric or colorectal cancer, MDPGCC was diagnosed in 54 (1.2\%). The baseline characteristics of the patients in the MDPGCC group are summarized in Table 1. The GP- and CP-MDPGCC groups comprised 24 (44.4\%) and 30 (55.6\%) patients, respectively. No statistically significant differences were observed between the two groups with regard to sex, age, operation, location or histological type. Twenty-three (93.3\%) in the GP-MDPGCC group were operated on for both gastric and colorectal cancer. Twenty-two (91.7\%) in the CP-MDPGCC group were operated on for both gastric and colorectal cancer. No differences were observed in rates of surgery between the two groups. No notable difference was observed in the year-by-year incidence of GP- and CP-MDPGCC as calculated from the date of surgery or chemotherapy for the secondary gastric or colorectal cancer (Figures 2(a) and (b)).

\subsection{Outcomes}

In the GP-MDPGCC group, death occurred in 1 case of gastric cancer (25.0\%) and 3 cases of colorectal cancer (75.0\%), while in the CP-MDPGCC group death occurred in 1 case of colorectal cancer (33.3\%) and 2 cases of gastric cancer (66.6\%).

\section{The Survival Curves of GP vs. CP-MDPGCC}

Overall survival rates in the two groups are shown in Figure 3. The 5-year survival rate in the GP- and CPMDPGCC groups was $84.7 \%$ and $83.3 \%$, respectively. No significant difference was observed between the GPand CP-MDPGCC groups $(\mathrm{P}=0.9)$.

\section{Discussion}

The results of this study revealed no difference in the prognosis between the GP- and CP-MDPGCC groups. According to some researchers, the incidence of multiprimary cancers in patients with gastric cancer was $1.1 \%-4.7 \%$
Table 1. Comparative data.

\begin{tabular}{|c|c|c|c|}
\hline Clinical features & $\begin{array}{c}\mathrm{GP} \\
\mathrm{n}=30(\%)\end{array}$ & $\begin{array}{c}C P \\
n=24(\%)\end{array}$ & $\mathrm{P}$ value \\
\hline \multicolumn{4}{|l|}{ Gender } \\
\hline Male & $23(76.6)$ & $17(70.8)$ & 0.63 \\
\hline Female & $7(23.3)$ & $7(29.2)$ & \\
\hline \multicolumn{4}{|l|}{ Age (Year) } \\
\hline Median [Range] & $70[45-81]$ & 68 [37-81] & \\
\hline \multicolumn{4}{|l|}{ Interval (Year) } \\
\hline Median [Range] & $6.1[1.0-22.8]$ & $5.2[1.1-23.1]$ & \\
\hline \multicolumn{4}{|l|}{ Operation } \\
\hline Both resection & 28 (93.3) & $22(91.7)$ & 0.81 \\
\hline Single resection & $2(6.7)$ & $2(8.3)$ & \\
\hline \multicolumn{4}{|l|}{ Location } \\
\hline Gastric (Upper) & $8(26.7)$ & $5(20.8)$ & 0.62 \\
\hline (Middle + Lower) & $22(73.3)$ & $19(79.2)$ & \\
\hline Colorectal (Colon) & $22(73.3)$ & $20(83.3)$ & 0.38 \\
\hline (Rectum) & $8(26.7)$ & $4(16.7)$ & \\
\hline \multicolumn{4}{|l|}{ Clinical Staging } \\
\hline Gastric (Stage I - III) & $29(96.7)$ & 21 (87.5) & 0.21 \\
\hline (Stage IV) & $1(3.3)$ & $3(12.5)$ & \\
\hline Colorectal (Stage I - III) & $26(86.7)$ & $23(95.8)$ & 0.25 \\
\hline (Stage IV) & $4(13.3)$ & $1(4.2)$ & \\
\hline \multicolumn{4}{|l|}{ Pathological Staging } \\
\hline Gastric (Stage I - III) & $27 \quad(90.0)$ & $21(87.5)$ & 0.23 \\
\hline (Stage IV) & $1(3.3)$ & $3(12.5)$ & \\
\hline Unknown & $2(6.7)$ & $0(0.0)$ & \\
\hline Colorectal (Stage I - III) & $27(90.0)$ & 21 (87.5) & 0.14 \\
\hline (Stage IV) & $3 \quad(10.0)$ & $0(0.0)$ & \\
\hline Unknown & $0(0.0)$ & $3(12.5)$ & \\
\hline \multicolumn{4}{|l|}{ Pathological type } \\
\hline Gastric (differentiated) & $15(55.6)$ & $15(68.2)$ & 0.37 \\
\hline (not differentiated) & $12(44.4)$ & $7(31.8)$ & \\
\hline Colorectal (differentiated) & $6(22.2)$ & $8(40.0)$ & 0.19 \\
\hline (not differentiated) & $21(77.8)$ & $12(60.0)$ & \\
\hline
\end{tabular}

PC = Gastric cancer precedence; GP = Colorectal cancer precedence. 


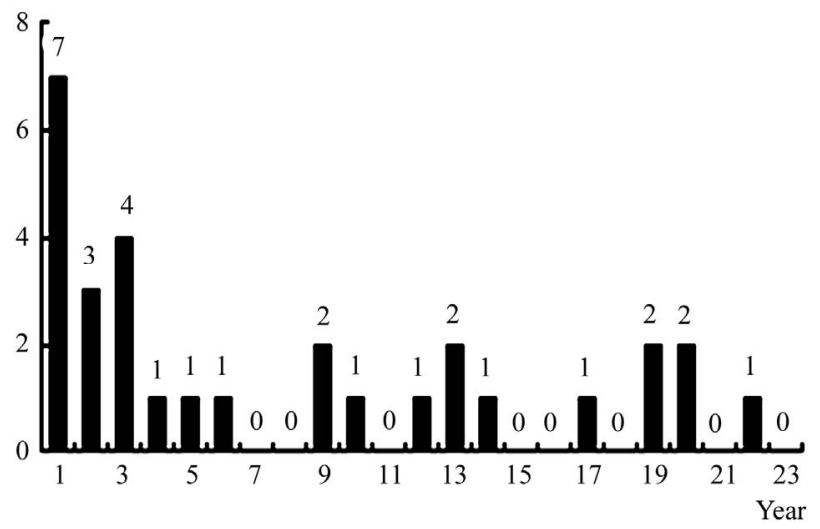

(a)

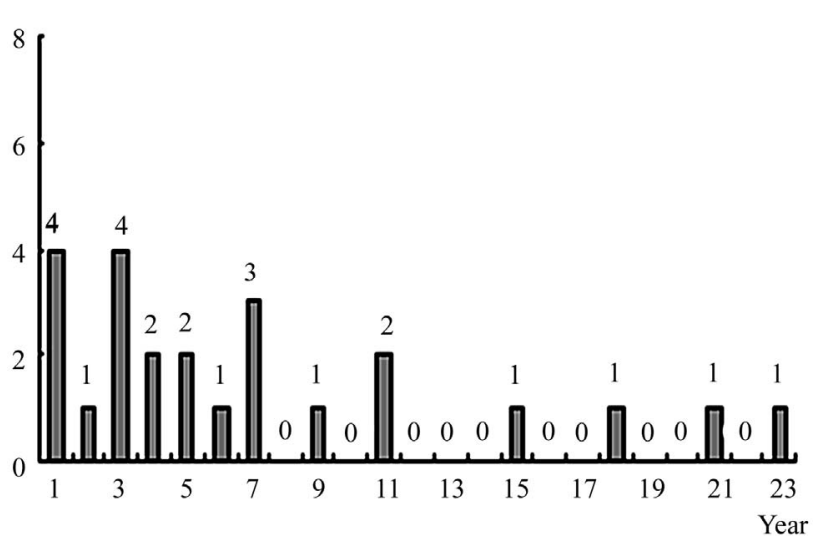

(b)

Figure 2. (a) Interval of GP-DPGCC between gastric and colorectal cancer; (b) Interval of CP-DPGCC between colorectal and gastric cancer.

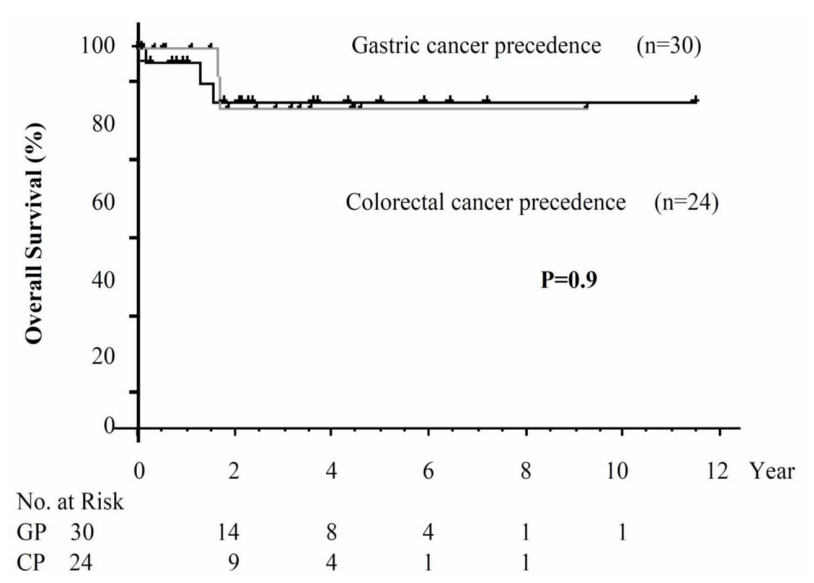

Figure 3. No difference was observed in survival rate between GP- and CP-MDPGCC groups, $P=0.9$.

[3-10], and the incidence of colorectal cancer in patients with gastric cancer was only about $1 \%$ [11]. In this study, the incidence of metachronous colorectal cancer in patients with gastric cancer was $1.2 \%$, which appears to be higher than that in previous studies involving no endoscopic resection and synchronous colorectal and gastric cancer. Patients with gastric or colorectal cancer may be at increased risk of developing metachronous gastric or colorectal cancer. A recent study has shown a rapidly increasing incidence of colorectal cancer in Japan [12]. And while the incidence of gastric cancer is slightly decreasing in Japan, the mortality rate of gastric cancer is the highest among malignant diseases. Remarkable advances in cancer treatment have resulted in great improvements in survival in many cancer patients. However, this means that these patients are at risk of developing DPGCCs and subsequently dying from another primary cancer. In earlier studies, the most common other primary cancer in patients with gastric cancer was colorectal cancer, followed by lung cancer, hepato-cellular-car- cinoma, renal-cell carcinoma and lymphoma [8,13-15]. The rapidly increasing rate of colorectal cancer in recent years has drawn special attention to this site in terms of SDPGCC and MDPGCC. However, to our knowledge, no reports have been published on the prognoses of GPand CP-MDPGCC. The results of this study demonstrated no difference in prognosis between GP- and CPMDPGCC. In an earlier study on the prognosis and clinicopathological features of SDPGCC and MDPGCC, we found that MDPGCC had a better prognosis than SDPGCC, and the proportion of non-advanced gastric and colorectal cancers was significantly higher in patients with MDPGCC [7]. The results of these earlier studies have raised the question as to which offers the best prognosis in MDPGCC, that with GP or CP. Therefore, the purpose of this study was to investigate the clinicopathological features of GP- and CP-MDPGCC and determine the biological significance of these two types of malignancy in making a prognosis. In this study, the 5-year survival rate in the GP- and CP-MDPGCC groups was $84.7 \%$ and $83.3 \%$, respectively. In our earlier study, it was suggested that the better prognosis observed with MDPGCC was due to more frequent detection in patients with early-stage cancer during routine follow-up after surgery for the first primary cancer. In fact, 54.5\% of patients with GP- and $50.0 \%$ of patients with CPDPGCC in the current analysis had received gastrointestinal examination before diagnosis of second primary cancer. In this study, death occurred from colorectal cancer as the second primary cancer in 3 cases (75.0\%) in the GP-, and from gastric cancer as the second primary cancer in 2 cases $(66.6 \%)$ in the CP-MDPGCC groups. These data appear to indicate a genetic and environmental relationship between gastric and colorectal cancer. Previous studies of patients receiving surgical treatment at all stages of gastric and colorectal cancer reported the 5-year survival rate to be $73.7 \%$ (11) and 69.9\% [16]. On 
the other hand, phase III clinical trials of S-1 plus cisplatin for advanced gastric cancer have yielded good responses and a median overall survival rate of 13.0 months [17]. Phase III clinical trials of FOLFOX4 for advanced colorectal cancer have yielded good responses and a median overall survival rate of 15.0 months [18]. Sensitivity of chemotherapy seems to be relationship between gastric and colorectal cancer. The genetic and environmental relationship between gastric and colorectal cancer may exert no influence on the prognosis of GP- or CP-MDPGCC. To our knowledge, this is the first study to report no difference in the prognosis for GP- and CP-MDPGCC. This indicates the importance of continuing periodic check-ups including screening for MDPGCC by gastrointestinal endoscopy over a long period of time. Since gastric and colorectal cancer patients may develop MDPGCC, effective postoperative diagnostic modalities need to be developed for the detection of both second primary gastric and colorectal cancers and the recurrence of primary gastric and colorectal cancers.

The goal of the present study was to determine which offered the better prognosis in MDPGCC, GP or CP. No significant difference was found in prognosis between GP- and CP-MDPGCC.

\section{Acknowledgements}

This work was mainly supported by a Grant-in-Aid for Scientific Research (A) 24249068 from the Ministry of Education, Culture, Sports, Science and Technology (MEXT), Japan.

\section{REFERENCES}

[1] W. Haenszel, F. B. Locke and M. Segi, “A Case-Control Study of Large Bowel Cancer in Japan," Journal of the National Cancer Institute, Vol. 64, No. 1, 1980, pp. 1722.

[2] B. Armstrong and R. Doll, "Environmental Factors and Cancer Incidence and Mortality in Different Countries, with Special Reference to Dietary Practices," International Journal of Cancer, Vol. 15, No. 4, 1975, pp. 617663. doi:10.1002/ijc.2910150411

[3] B. W. Eom, H.-J. Lee, M.-W. Yoo, J. J. Cho, W. H. Kim, H.-K. Yang and K. U. Lee, "Synchronous and Metachronous Cancers in Patients with Gastric Cancer," Journal of Surgical Oncology, Vol. 1, No. 98, 2008, pp. 106-110. doi:10.1002/jso.21027

[4] Y. Ikeda, M. Saku, H. Kawanaka, M. Nonaka and K. Yoshida, "Features of Second Primary Cancer in Patients with Gastric Cancer,” Oncology, Vol. 65, No. 2, 2003, pp. 113-117. doi:10.1159/000072335

[5] Y. Maehara, S. Tomisaki, Y. Emi, Y. Sakaguchi, T. Kusumoto, Y. Ichiyoshi and K. Sugimachi, "Clinicopathological Features of Patients Who Died with Second
Primary Cancer after Curative Resection for Gastric Cancer,” Anticancer Research, Vol. 15, No. 3, 1995, pp. 1049-1053.

[6] H. Furukawa, M. Hiratsuka, T. Iwanaga, S. Imaoka, T. Kabuto, O. Ishikawa, Y. Sasaki, M. Kameyama, H. Ohigashi and S. Nakamori, "Treatments for Second Malignancies after Gastrectomy for Stomach Cancer," Hepatogastroenterology, Vol. 43, No. 7, 1996, pp. 194-198.

[7] M. Watanabe, M. Kochi, M. Fujii, T. Kaiga, Y. Mihara, T. Funada, H. Tamegai, H. Shimizu and T. Takayama, "Double Primary Gastric and Colorectal Cancer: Is the Better Prognosis Synchronous or Metachronous?” American Journal of Clinical Oncology, Vol. 35, No. 5, 2012, pp. 407-410. doi:10.1097/COC.0b013e318218585a

[8] C. W. Wu, S. S. Lo, J. H. Chen, M. C. Hsieh, A. F. Li and W. Y. Lui, "Multiple Primary Cancers in Patients with Gastric Cancer ” Hepatogastroenterology, Vol. 53, No. 69, 2006, pp. 463-467.

[9] G. Lundegårdh, L. E. Hansson, O. Nyrén, H. O. Adami and U. B. Krusemo, "The Risk of Gastrointestinal and Other Primary Malignant Diseases Following Gastric Cancer ” Acta Oncologica, Vol. 30, No. 1, 1991, pp. 1-6. doi:10.3109/02841869109091804

[10] J. H. Lee, J. S. Bae, K. W. Ryu, J. S. Lee, S. R. Park, C. G. Kim, M. C. Kook, I. J. Choi, Y. W. Kim, J. G. Park and J. M. Bae, "Gastric Cancer Patients at High-Risk of Having Synchronous Cancer," World Journal of Gastroenterology, Vol. 28, No. 12, 2006, pp. 2588-2592.

[11] M. Ikeguchi, S. Ohfuji, A. Oka, S. Tsujitani, M. Maeda and N. Kaibara, "Synchronous and Metachronous Primary Malignancies in Organs Other than the Stomach in Patients with Early Gastric Cancer," Hepatogastroenterology, Vol. 42, No. 5, 1995, pp. 672-676.

[12] JSCCR, “Japanese Society for Cancer of the Colon and Rectum (JSCCR) Colon and Rectum Treatment Guidelines,” Kanehara \& Co. Ltd., Tokyo, 2009.

[13] S. Saito, Y. Hosoya, K. Togashi, K. Kurashina, H. Haruta, M. Hyodo, K. Koinuma, H. Horie, Y. Yasuda and H. Nagai, "Prevalence of Synchronous Colorectal Neoplasms Detected by Colonoscopy in Patients with Gastric Cancer,” Surgery Today, Vol. 38, No. 1, 2008, pp. 20-25.

[14] N. Kaibara, M. Maeta and M. Ikeguchi, "Patients with Multiple Primary Gastric Cancers Tend to Develop Second Primaries in Organs Other than the Stomach," Surgery Today, Vol. 23, No. 2, 1993, pp. 186-188. doi:10.1007/BF00311241

[15] M. Ueno, T. Muto, M. Oya, H. Ota, K. Azekura and T. Yamaguchi, "Multiple Primary Cancer: An Experience at the Cancer Institute Hospital with Special Reference to Colorectal Cancer," International Journal of Clinical Oncology, Vol. 8, No. 3, 2003, pp. 162-167. doi:10.1007/s10147-003-0322-z

[16] Japanese Gastric Cancer Association, “Japanese Gastric Cancer Treatment Guideline 2010 (ver. 3),” Gastric Cancer, Vol. 14, No. 2, 2011, pp. 113-123. doi:10.1007/s10120-011-0042-4

[17] W. Koizumi, H. Narahara, T. Hara, A. Takagane, T. 
Akiya, M. Takagi, K. Miyashita, T. Nishizaki, O. Kobayashi, W. Takiyama, Y. Toh, T. Nagaie, S. Takagi, Y. Yamamura, K. Yanaoka, H. Orita and M. Takeuchi, "S-1 plus Cisplatin versus S-1 Alone for First-Line Treatment of Advanced Gastric Cancer (SPIRITS trial): A Phase III Trial,” The Lancet Oncology, Vol. 9, No. 3, 2008, pp. 215-221. doi:10.1016/S1470-2045(08)70035-4

[18] G. Colucci, V. Gebbia, G. Paoletti, F. Giuliani, M. Caruso, N. Gebbia, G. Cartenì, B. Agostara, G. Pezzella, L. Manzione, N. Borsellino, A. Misino, S. Romito, E. Durini, S. Cordio, M. Di Seri, M. Lopez, E. Maiello, S. Montemurro,
A. Cramarossa, V. Lorusso, M. Di Bisceglie, M. Chiarenza, M. R. Valerio, T. Guida, V. Leonardi, S. Pisconti, G. Rosati, F. Carrozza, G. Nettis, M. Valdesi, G. Filippelli, S. Fortunato, S. Mancarella, C. Brunetti and Gruppo Oncologico Dell'Italia Meridionale, "Phase III Randomized Trial of FOLFIRI versus FOLFOX4 in the Treatment of Advanced Colorectal Cancer: A Multicenter Study of the Gruppo Oncologico Dell'Italia Meridionale,” Journal of Clinical Oncology, Vol. 1, No. 23, 2005, pp. 48664875. doi:10.1200/JCO.2005.07.113 